\section{IN BRIEF}

\section{PAEDIATRIC RHEUMATOLOGY}

\section{Lung disease in SJIA has distinct features}

Systemic juvenile idiopathic arthritis (sJIA)-associated lung disease is distinct from other inflammatory lung conditions, according to the results of new study. In patients with sJIA attending the Cincinnati Children's Hospital Medical Center, the presence of lung disease was associated with a young age of diagnosis, a history of macrophage activation syndrome and prior adverse reactions to cytokine-targeted biologic therapy. Lung disease in these patients shared some histopathological features with pulmonary alveolar proteinosis but had different immunological features, including increased IL-18.

ORIGINAL ARTICLE Schulert, G. S. et al. Systemic juvenile idiopathic arthritis-lung disease: characterization and risk factors. Arthritis Rheumatol. https://doi.org/10.1002/ art.41073 (2019)

\section{$\Rightarrow$ SYSTEMIC SCLEROSIS}

\section{Protecting against myocardial disease in SSc}

In a prospective observation study of 601 patients with systemic sclerosis (SSc), the use of vasodilators (including calcium channel blockers, angiotensin-converting enzyme (ACE) inhibitors and/or angiotension II receptor blockers) was associated with a reduced incidence of ventricular arrhythmias (hazard ratio (HR) $0.28,95 \% \mathrm{Cl} 0.09-0.90$ ). In the same multi-variant Cox regression analysis, low-dose acetylsalicylic acid (ASA) $(\leq 325 \mathrm{mg}$ daily) was associated with a reduced incidence of cardiac blocks and/or $\mathrm{Q}$ waves and/or pacemaker implantation (HR 0.46, 95\% Cl 0.24-0.87). ORIGINAL ARTICLE Valentini, G. et al. Vasodilators and low-dose acetylsalicylic acid are associated with a lower incidence of distinct primary myocardial disease manifestations in systemic sclerosis: results of the DeSScipher inception cohort study. Ann. Rheum. Dis. https://doi.org/10.1136/annrheumdis-2019-215486 (2019)

\section{$\Rightarrow$ GENETICS}

\section{Two GWAS loci identified in IgG4-related disease}

The first genome-wide association study (GWAS) of lgG4-related disease, involving 857 Japanese patients with lgG4-related disease and 2,082 healthy participants, has identified two susceptibility loci: HLA-DRB1 and FCGR2B. The strongest disease association in HLA-DRB1 corresponded to an amino acid residue in the peptide-binding groove of HLA-DRB1. The single nucleotide variant in FCGR2B (rs1340976) was associated with increased expression of FCGR2B, as well as with specific clinical features of lgG4-related disease (including the number of swollen organs and $\lg 44$ concentration at diagnosis).

ORIGINAL ARTICLE Terao, C. et al. IgG4-related disease in the Japanese population: a genome-wide association study. Lancet Rheumatol. https://doi.org/10.1016/ S2665-9913(19)30006-2 (2019)

\section{$\Rightarrow$ THERAPY}

\section{Pregnancy outcomes in patients with JIA}

Registry data from a long-term observation study of patients with juvenile idiopathic arthritis (JIA) suggest that DMARD exposure does not increase the risk of major adverse pregnancy outcomes in patients with JIA. Among the 152 pregnancies in 98 women with JIA and 39 pregnancies involving men with JIA as partners, the rates of miscarriage (13.1\%) and major congenital anomaly $(3.6 \%)$ were similar to the expected background rates. Half of the pregnancies were unplanned and occurred during treatment with DMARDs. Elective abortions were also common in DMARD-exposed pregnancies.

ORIGINAL ARTICLE Drechsel, P. et al. Pregnancy outcomes in DMARD-exposed patients with juvenile idiopathic arthritis—results from a JIA biologic registry. Rheumatology. https://doi.org/10.1093/rheumatology/kez309 (2019)

\title{
Vaccination guidance updated
}

Prevention of infection is important in the management of autoimmune inflammatory rheumatic diseases (AIIRD), but uptake of vaccinations is suboptimal in patients with AIIRD worldwide. In light of new data on the prevalence and incidence of vaccine-preventable infections in adults with AIIRD, as well as on the efficacy, immunogenicity and safety of available vaccines, EULAR has issued updated recommendations for vaccinations in these patients.

The 2019 update comprises six overarching principles and nine recommendations, formulated by an international group of experts and based on a comprehensive systematic literature review. "Since the first version of EULAR recommendations on vaccination of adult patients with AIIRD was published in 2011, there has been a large expansion in the amount of available evidence on this topic, necessitating an update," says lead author Victoria Furer.

Notably, the EULAR task force used clear outcome measures of vaccination when evaluating this evidence. "In the AIIRD population, the data on the clinical efficacy of vaccination is limited," explains Furer. "Thus, 'immunogenicity' of vaccination was used as a surrogate marker of efficacy, when appropriate. The strength of recommendations was based on the level of the data. For example, in case of lack of a direct correlation between the immunogenicity outcomes and the level of protection, the strength of recommendation was downgraded."

The overarching principles stress the need for regular assessment, patient education and shared decision-making. "We hope that this recommendation will improve the implementation of the vaccination programme," notes Furer. The principles also state that vaccines should be administered during quiescent disease and before planned immunosuppressive therapy, and that nonlive vaccines can be given to patients being treated with glucocorticoids and/or DMARDs. Live-attenuated vaccines should be avoided during immunosuppression but, in a modification of the 2011 recommendations, MMR and herpes zoster vaccines can be considered with caution.

The core set of recommendations concerning influenza, pneumococcal, tetanus toxoid, hepatitis A, hepatitis B and $\mathrm{HPV}$ vaccinations remained essentially unchanged from the 2011 recommendations, with some minor modifications. Several of the 2011 recommendations were omitted from the 2019 update; two, concerning BCG vaccination and vaccination of hyposplenic or asplenic patients with AIIRD, had become irrelevant to clinical practice and one, concerning travelling patients, was deemed non-specific. New recommendations were added to encourage the vaccination of immunocompetent members of the households of patients with AIIRD, to avoid vaccination with liveattenuated vaccines for the first 6 months of life in newborns exposed to biologic drugs during the late stages of pregnancy and to avoid vaccination against yellow fever in patients with AIIRD during immunosuppression.

"The implementation of the present recommendations will help in prevention of infections in the susceptible population of patients with AIIRD," Furer contends. "In particular, dissemination of the main principles of these recommendations among the health professionals, including primary care teams, treating patients with AIIRD is of great importance. Thus, a number of educational projects for the medical community to increase the awareness of vaccination and compliance with the recommendations are underway."

Sarah Onuora

ORIGINAL ARTICLE Furer, V. et al. 2019 update of EULAR recommendations for vaccination in adult patients with autoimmune inflammatory rheumatic diseases. Ann. Rheum. Dis. https:// doi.org/10.1136/annrheumdis-2019-215882 (2019) 